Pla Sirvent, Dimas. Turismo y política: cómo afectan los grandes eventos al territorio. GeoGraphos. [En línea]. Alicante: Grupo Interdisciplinario de Estudios Críticos y de América Latina (GIECRYAL) de la Universidad de Alicante, 18 de enero de 2013, vol. 4, n 36, p. 93-116. [ISSN: 2173-1276].

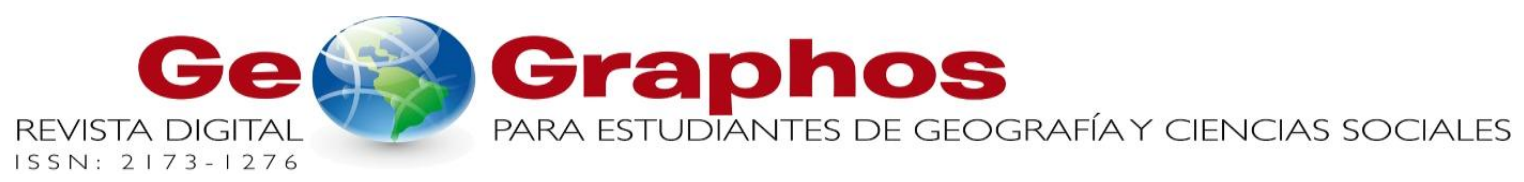

<http://web.ua.es/revista-geographos-giecryal $>$

Vol. 4. No 36

Año 2013

\title{
TURISMO Y POLÍTICA: CÓMO AFECTAN LOS GRANDES EVENTOS AL TERRITORIO
}

\author{
Dimas Pla Sirvent \\ Estudiante de la Licenciatura de Geografía \\ Facultad de Filosofía y Letras. Universidad de Alicante (Alicante, España) \\ Correo electrónico: dps12@alu.ua.es/dimas.plasir@gmail.com
}

Recibido: 21 de junio de 2012. Devuelto para revisión: 15 de septiembre de 2012. Aceptado: 18 de enero de 2013

\section{RESUMEN}

En la España actual y en la crisis tan profunda que existe hoy en día, es necesario mirar hacia atrás y buscar el error o errores en su conjunto. Las claves para desengranar la repercusión de la política en el turismo y en como afecta esta política en la celebración de grandes eventos turísticos. Hay que indagar y reflexionar sobre las claves que producen este fenómeno, como el coste económico que tienen en la situación actual, y si es lícito este gasto pudiendo invertir ese dinero en obras sociales necesarias. Además hay que profundizar en los costes sociales y cómo repercute esta política sobre la población y el territorio.

Palabras clave: Política turística, Coste económico, Sociedad, Estado del bienestar. 


\title{
TOURISM AND POLITICS: HOW THE MAJOR EVENTS AFFECTING THE TERRITORY
}

\begin{abstract}
In present-day Spain and the deep crisis that exists these days, it's necessary to look back and try to find the mistake or mistakes as a whole. The clues to take out of gear the impact of the policy in the tourism and how it affects this policy in the celebration of big touristic events. It must investigate and think about the clues that produce this phenomenon, like the economic cost that it have in the actual situation, and if it's legal this cost it may invest this money in necessaries benevolent works. Apart from that, it must to go into the social costs and how it have repercussions this policy on the population and the region.
\end{abstract}

Key words: Tourism policy, Economic cost, Society, State welfare.

\section{TURISMO E POLÍTICA: COMO OS EVENTOS PRINCIPAIS QUE AFECTAN O TERRITÓRIO}

\section{RESUMO}

A Espanha atual e a profunda crise que existe hoje, é necessário olhar para trás e encontrar o erro ou erros como um todo. Chaves para desengatar o impacto da política de turismo e como afeta a esta política em eventos turísticos principais. Você deve investigar e refletir sobre as chaves que produzem esse fenômeno, como o custo econômico da situação atual, e se esta despesa pode investir esse dinheiro em trabalho social necessário é legal. Devemos também aprofundar os custos sociais e como os impactos desta política na população e território.

Palavras-chave: Política de Turismo, custo económico, sociedade, bem-estar social.

\section{INTRODUCCIÓN}

Para poder analizar la situación actual es necesario realizar una evolución sobre la política turística del siglo XX, en la cual existen tres etapas muy diferenciadas y en la que se realizan políticas diferentes y repercutiendo en el turismo. Esta evolución se dividirá en tres etapas: la dictadura de Alfonso XIII y la II Republica, la dictadura de Franco y la democracia dividiendo la etapa socialista de la etapa del Partido Popular. Es evidente que realmente lo que interesa es analizar la situación actual en profundidad y encontrar las claves que producen este fenómeno de los grandes eventos, para que sirven y su repercusión sobre la sociedad y sobre el territorio. Para llegar a este punto hay que atender y comprender a lo acontecido a la repercusión política en el turismo durante el siglo XX. ¿Estrategia electoral? ¿Beneficio personal de los propios políticos? Estas dos cuestiones y otras se intenta darle respuesta a lo largo del desarrollo del trabajo. A lo largo del trabajo vamos a tener el inconveniente de que datos exactos de los costes que han supuesto los grandes eventos no los vamos a poder aportar, por su imposibilidad de conseguirlos, ya que el Partido Popular se niega hacerlos públicos. Esto será otra de las claves en la que nos adentraremos y por la cual nos podemos preguntar ¿Esconden algo? ¿Hay ilegalidades en los procedimientos? Evidentemente podemos dar claves que algo de esto hay, y a las pruebas me remito, de toda la trama de corrupción que acecha a nuestra 
comunidad autónoma, es evidente y se ha demostrado que detrás de la organización de estos grandes eventos hay muchas de las ilegalidades que han salido a la luz.

Por todas estas razones nos invitan a investigar e intentar explicar de la mejor manera posible la problemática que nos concierne en la comunidad valenciana y la repercusión de la política en el turismo y más concretamente en la celebración de grandes eventos, que según nos venden algunos políticos que están involucrados con ello, su fin por encima de todo es promocionar las ciudades valencianas por todo el mundo. Pero esto habrá que ponerlo en una balanza a ver hacia donde se desequilibra.

\section{EVOLUCIÓN HISTÓRICA DE LAS REPERCUSIONES DE LA POLÍTICA ESPAÑOLA SOBRE EL TURISMO DESDE EL SIGLO XX HASTA LA ACTUALIDAD}

Para poder analizar la evolución histórica de la repercusión política en el turismo hay que remontarse al año 1905 que fue cuando se inició la organización administrativa del turismo en España.

En función de los diferentes modelos de política turística aplicados por la Administración española a lo largo del siglo XX, hemos dividido la evolución histórica en tres grandes apartados. En el primero de ellos se estudia la intervención del Estado entre 1900 y 1936, es decir, el periodo en que tiene lugar la emergencia del turismo moderno en España. En el segundo apartado se analiza la política turística durante los años de la dictadura franquista, cuando el turismo dejó de ser algo minoritario y se transformó en un fenómeno de masas. Por último, el análisis se centra en lo acontecido en el último cuarto del siglo $\mathrm{XX}$, donde destacan la definitiva consolidación de España como destino turístico de primer orden y la responsabilización de competencias de turismo por parte de las Comunidades Autónomas.

\section{La monarquía de Alfonso XIII y la II República (1902-1936)}

A lo largo de las dos primeras décadas del siglo XX, y por encima del carácter liberal o conservador de los gobiernos de turno, en la escena económica española primó el proteccionismo y la intervención estatal. Y el turismo, a pesar de ser todavía una actividad muy minoritaria en nuestro país, no quedó al margen de dicha intervención. Las autoridades monárquicas españolas, conscientes de que la escasa presencia de visitantes extranjeros nos estaba privando de unas divisas vitales para la buena marcha de nuestra economía, consideraron que la Administración debía fomentar y controlar las actividades turísticas. El principal objetivo de su política turística fue la máxima captación de turistas extranjeros. Deseo que, como se verá más adelante, no varió un ápice ni durante la dictadura de Primo de Rivera ni en la España republicana. Una de la primeras actuaciones acometidas fue la de fomentar en nuestro país las excursiones artísticas y de recreo del público extranjero. La Comisaría Regia del Turismo y Cultura Artística (constituida en 1911) en sus inicios se preocupó, fundamentalmente, de promocionar España en el extranjero y de incrementar y cuidar el patrimonio cultural español. A pesar de la escasa ayuda oficial se crearon museos, se construyeron jardines, se organizaron exposiciones de arte, se conservaron monumentos, se fundaron bibliotecas y archivos, y se editó un buen número de excelentes publicaciones en las que los turistas podían encontrar desde posibles itinerarios para conocer nuestra geografía, hasta estudios pormenorizados de la riqueza artística de diversas ciudades monumentales españolas. 
Durante los años de la dictadura de Primo de Rivera se prolongaron las prácticas proteccionistas y de intervención estatal de las décadas precedentes. Respecto a la intervención política de la Dictadura de Primo de Rivera las autoridades del Directorio Militar decidieron que la Comisaría Regia siguiera al frente de la organización administrativa del turismo y, desgraciadamente, en las mismas condiciones. Es decir, continuó siendo un órgano escasamente dotado y excesivamente personalizado.

Pero el Comisario Regio no era la única persona que a mediados de la década de los años veinte consideraba que el turismo merecía más atención oficial. Existía una creciente corriente de opinión que preconizaba la creación de un nuevo organismo estatal que, dotado de mayores recursos, fuera capaz de atender eficazmente a los variados aspectos que se relacionaban con el turismo: carreteras, ferrocarriles, navegación, hoteles y propaganda, entre otros. Con esto podemos observar un reducto del pensamiento que años más tarde se realizaría en nuestro país, pero en esta época solo era un ligero pensamiento de una persona, que las autoridades se hicieron eco de esa solicitud y por Real Decreto de 25 de abril de 1928 crearon el Patronato Nacional del Turismo. Este nuevo órgano contó con mayores medios humanos y económicos, si bien estos últimos continuaron siendo considerados por los responsables turísticos como insuficientes, y tuvo que acometer una tarea bastante más amplia de la que hasta ese momento había venido llevando a cabo la Comisaría Regia: la preparación de España para el turismo y la propaganda de nuestro país, dentro y fuera de su territorio, para estimular el turismo interior y atraer el extranjero. Para ello, y a diferencia de lo que había sucedido con la Comisión Nacional y la Comisaría Regia, que se habían financiado gracias a las dotaciones presupuestarias incluidas en las correspondientes al Ministerio de Fomento y al Ministerio de Instrucción Pública y Bellas Artes, respectivamente, se estipuló que la financiación del Patronato Nacional corriera a cargo de los recursos que se obtuvieran con la creación de un seguro obligatorio de las personas que viajaran por ferrocarril y, también, del ganado vivo transportado en él. Además, se señaló que hasta que dicho seguro entrara en funcionamiento el Tesoro anticiparía al Patronato, con cargo reintegrable, la cantidad máxima de 250.000 pesetas, es decir, aproximadamente la mitad de todo el dinero que había recibido la Comisaría Regia durante el periodo 1924-1927.

Con la creación del Patronato Nacional del Turismo, e incluso durante los últimos años de existencia de la Comisaría Regia, las autoridades introdujeron un notable cambio en la política turística española, que hasta ese momento se había caracterizado por la escasez de medios aplicados y por una excesiva concentración en la promoción del país y en el arte como reclamo turístico. La gran novedad no se cifró en el aspecto económico, ya que, a juicio de los 271 responsables turísticos los independientes y crecientes recursos de los que dispuso el Patronato continuaron siendo escasos e insuficientes, sino en que por vez primera la Administración española actuó sobre un amplio abanico de actividades estrechamente relacionadas con el turismo. Se consideró prioritario de cara al desarrollo del turismo, y especialmente ante la prevista celebración en 1929 de las exposiciones internacionales de Sevilla y Barcelona, ampliar y mejorar la infraestructura hotelera; crear un servicio que, tanto en el interior como en el exterior del país, informara amplia y verazmente sobre nuestros atractivos turísticos; incrementar nuestros sistemas de transporte y la calidad de los mismos; y fomentar las actividades artísticas, deportivas y festivas, así como la celebración de congresos.

En canto a la la labor desarrollada por la oferta hotelera merece la pena destacar la creación de:

1) El Servicio de Crédito Hotelero, con la finalidad de estimular y auxiliar la construcción de hoteles por parte de la iniciativa privada.

2) El Título de Establecimiento Recomendado, como estímulo para el mejoramiento de las industrias relacionadas con el turismo, y especialmente la hotelera, y como garantía para el viajero. 
3) La Cámara Oficial Hostelera, con la misión de perseguir la clandestinidad, evitar los abusos y organizar la industria del hospedaje.

4) La Guía Oficial, con el objetivo de proporcionar a los viajeros una relación de alojamientos y de sus precios.

5) El Libro Oficial de Reclamaciones.

6) La Red de Paradores y Albergues de Carretera, una oferta hotelera pública caracterizada por precios ajustados, servicios de calidad e instalaciones confortables que se ubicarían en áreas turísticamente atractivas pero con una escasa o inexistente infraestructura hotelera privada.

Por otro lado, el Patronato Nacional abrió al público siete agencias informativas en el extranjero y alrededor de medio centenar en el territorio español y, al igual que la Comisaría Regia, hizo un valioso esfuerzo editorial de cara a la divulgación turística de nuestro país. Asimismo, sabemos que durante la Dictadura de Primo de Rivera se prestó una atención especial al aumento y mejora de nuestros sistemas de transporte. Se mejoró la red ferroviaria y se renovó y amplió el material móvil utilizado en la misma. Además, el 9 de febrero de 1926 se creó el Circuito Nacional de Firmes Especiales, que permitió en tres años y medio la modernización de 2.800 kilómetros de carreteras. Tampoco hay que olvidar que se avanzó significativamente en el todavía novedoso transporte aéreo. Lógicamente, todo esto fue muy positivo de cara al turismo. También lo fue el hecho de que el Patronato Nacional negociara y consiguiera que algunas compañías de transportes terrestre y marítimo contribuyeran, mediante la concesión de determinadas facilidades a sus clientes, a incentivar el movimiento de viajeros. Por último, conviene reseñar que, al margen de los eventos de Sevilla y Barcelona, las autoridades utilizaron como motivos de atracción turística las actividades artísticas (exposiciones) y deportivas (golf, regatas, hípicas y motorismo), las fiestas populares (Valencia, Castellón, Alicante, San Sebastián) y la organización de congresos.

Tras la proclamación de la República, el Patronato Nacional del Turismo experimentó notables cambios, producidos por la etapa convulsa que vivía el país en esa etapa y que iría en aumento con forme pasaban los años y con lo que venía a continuación. Y la mejor prueba de ello es que hay poco que destacar de la política turística republicana. Si acaso, las labores de promoción y propaganda, el incremento registrado en la Red de Paradores y Albergues de Carretera, que en 1936 contaba ya con 15 establecimientos, y el espaldarazo dado a la Federación Española de Sindicatos de Iniciativas y Turismo al declararla de utilidad pública por Orden de 21 de noviembre de 1935.

\section{La dictadura de Franco (1939-1975)}

Como es lógico, el inicio de la Guerra Civil paralizó la actividad turística del país por completo, es decir, la llegada de turistas se convirtió en nula. Poco después de terminar la guerra civil se empezaron a tomar medidas para reactivar la política turística La que fue aplicada a lo largo de la difícil década de los años cuarenta fue, como no podía ser de otro modo, claramente intervencionista. El primer paso en este sentido se dio apenas una semana después de que finalizara la Guerra Civil. Con la Orden de 8 de abril de 1939 la Administración trató de intensificar su control sobre la industria hotelera y mejorar el funcionamiento de la misma. A partir de esta normativa serían las autoridades competentes en la materia las encargadas de autorizar la apertura de establecimientos hoteleros y de fijar las categorías de dichos locales y los precios máximos y mínimos exigidos en los 
mismos. Las autoridades franquistas entendieron que el turismo podía ser uno de los pilares donde debería apoyarse el desarrollo económico del país. Las divisas que el turismo exterior podría aportar se consideraron imprescindibles para la recuperación económica de la nación tras la guerra civil. Por lo tanto, el control de precios se perfiló como una medida trascendental. Y buena prueba de ello es que los precios que la Administración fijó en el año 1941 para el alojamiento, la pensión completa y el cubierto obligatorio, no experimentaron alteración alguna hasta el año 1947.

Del resto de la política turística merece la pena destacar:

1) La reorganización de las Juntas Provinciales y Locales, para desarrollar una misión similar a la que habían venido desarrollando los sindicatos de iniciativas, muchos de ellos desaparecidos durante la guerra civil.

2) La reglamentación de la publicidad con fines de propaganda turística y de la publicidad de cualquier orden cuando los lugares en que se situaran los anuncios ofrecieran interés desde el punto de vista turístico.

3) La creación definitiva del Crédito Hotelero, que tendría como finalidad estimular y auxiliar la construcción e instalación de hoteles adecuados, o similares, en aquellas poblaciones y lugares que la Dirección General de Turismo juzgase de interés nacional o turístico, y facilitar la transformación y mejora de los ya existentes.

4) La instauración con fines recaudatorios de la Póliza de Turismo.

5) La regulación del ejercicio de las actividades mercantiles de las agencias de viajes.

6) El reconocimiento del Sindicato Nacional de Hostelería y Similares como la única organización con personalidad suficiente para la representación y disciplina de los intereses de la producción en esta rama de la economía.

7) La protección de los intereses de la red pública de establecimientos al prohibir la utilización de los términos "Parador de Turismo", "Albergue de Turismo", "Hostería de Turismo" y "Refugio de Turismo" a las empresas privadas dedicadas a la industria de hospedaje o a servicios de comidas".

8) La construcción y explotación de la red estatal de establecimientos, que alcanzó en el año 1951 la cifra de 26 centros.

9) La aprobación del Reglamento Nacional de Trabajo para la Industria Hotelera y de Cafés, Bares y Similares y del reglamento Ordenador de los Transportes por Carretera.

10) La creación, en el seno del Instituto Nacional de Industria, de la empresa Autotransporte Turístico Español, con la que se quiso incrementar la oferta de transporte por carretera.

En el seno de la dictadura de Franco, se debe destacar el período que se extiende entre 1951 y 1962. Esta etapa pone fin al aislamiento económico y político de la España de Franco, el progresivo, aunque algo lento, abandono de la política autárquica y el Plan de Estabilización de 1959, la política turística fue, en líneas generales, bastante continuista respecto a la practicada en los años cuarenta. La Administración siguió siendo poco generosa con el turismo, tanto por lo que respecta a las dotaciones presupuestarias como al crédito hotelero, y continuó regulando e interviniendo en el sector: 
1) Se obliga a los hoteles a exhibir las listas de precios.

2) Se aprueba el Reglamento para el ejercicio de las profesiones libres de Guías, Guías-Intérpretes y Correos de Turismo.

3) Se regula el alojamiento de turistas en casas particulares.

4) Se amplia y modifica el reglamento sobre transporte de viajeros por carretera realizado por las agencias de viajes.

5) Se prohíbe que cuando en el interior de una población exista un Albergue o Parador de Turismo, se instale a menos de 10 kilómetros del mismo, en las carreteras o caminos que a ella afluyan, un establecimiento hotelero privado.

6) Se reglamentan los campamentos de turismo y las actividades de la hostelería y de las agencias de viajes.

La última etapa de la dictadura franquista la podemos denominar como los "años del desarrollo", entendiendo como tales la década de los años sesenta y el quinquenio siguiente, es decir, hasta la muerte de Franco, las competencias turísticas continuaron correspondiendo al Ministerio de Información y Turismo. En el mes de julio de 1962 Manuel Fraga fue nombrado nuevo Ministro. Poco después, en el mes de septiembre, se crearon la Subsecretaría de Turismo, la Dirección General de Promoción del Turismo y la Dirección General de Empresas y Actividades Turísticas. Esta estructura organizativa sólo experimentó un cambio durante el periodo analizado. La Subsecretaría de Turismo fue suprimida por razones económicas desde noviembre de 1967 a noviembre de 1974.

Pero a lo que nuestro estudio nos centra la política turística practicada durante el periodo 1962-1975, enmarcada, como es natural, dentro de la denominada planificación indicativa, tuvo como objetivo prioritario conseguir un crecimiento máximo. El país necesitaba divisas, el turismo las podía proporcionar, y se consideró que lo más conveniente era crecer cuanto más mejor, tanto en término de demanda como de oferta. Sin embargo, este objetivo maximizador se vio acompañado de una insuficiente asignación de recursos. La participación del Ministerio de Información y Turismo en los presupuestos generales del Estado fue siempre muy reducida. También lo fueron las inversiones públicas en turismo previstas y ejecutadas en los tres planes de desarrollo que vieron la luz, así como la política de crédito oficial. No puede decirse, pues, que el Estado fuera especialmente generoso con el turismo. La intervención del Estado se centró fundamentalmente sobre la oferta y, de manera muy especial, sobre tres grupos de objetivos:

1) Control de precios y calidad de los servicios.

2) Fomento de la capacidad de alojamiento.

3) Organización administrativa. Por lo que respecta al control de los precios y de la calidad de los servicios turísticos la legislación fue abundante.

De ella habría que destacar:

1) Determinación de precios a percibir por la industria hotelera y en los campamentos de turismo. 
2) Actualización y reajuste de los honorarios de los Guías y Guías-Intérpretes.

3) Fijación de los precios máximos del plato combinado turístico y del menú turístico.

4) Regulación de las condiciones de sanidad, seguridad y habitabilidad en apartamentos.

5) Requisitos mínimos en infraestructura en los alojamientos turísticos.

6) Tenencia del Libro de Reclamaciones.

7) Imposición de multas y sanciones.

8) Exigencia de graduación para desempeñar tareas directivas en los establecimientos turísticos, incluidos los de propiedad estatal.

En cuanto al fomento de la capacidad de alojamiento, las actuaciones del Estado se centraron en:

1) Crédito hotelero.

2) Préstamos para financiar la construcción y venta de edificaciones para extranjeros en zonas turísticas.

3) Inversión en estaciones invernales de montaña.

4) Primer Plan de Modernización Hotelera.

5) Créditos para la financiación de capital circulante de las empresas turísticas

6) Inversión directa del Estado a través de la red estatal de paradores, que continuó creciendo durante el periodo, y de las empresas públicas ATESA y ENTURSA, creada esta última en el año 1963 con el objetivo de construir y explotar establecimientos hoteleros.

De la amplia legislación sobre la organización administrativa de la oferta hay que destacar:

1) Reglamento de las actividades turístico informativas privadas.

2) Registro de Denominaciones Geoturísticas.

3) Registro de Empresas y Actividades Turísticas.

4) Ordenación de restaurantes y creación del menú turístico.

5) Régimen de reservas hoteleras

6) Estatuto Ordenador de las Empresas y de las Actividades Turísticas Privadas.

7) Ordenación de cafeterías, campamentos de turismo, apartamentos y otros alojamientos similares. 
8) Clasificación de los establecimientos hoteleros.

9) Ordenación turística de las Ciudades de Vacaciones.

10) Estatuto de los Directores de Empresas Turísticas.

11) Determinación de zonas y rutas turísticas.

12) Ordenación del Trabajo para la industria hostelera.

13) Ordenación de la oferta turística.

La Administración trató también de fomentar la oferta turística a través de la formación de mano de obra, de la ordenación espacial de la oferta y de la conservación y desarrollo de los recursos turísticos y de las infraestructuras:

1) Creación de la Escuela Oficial de Turismo.

2) Ley de Centros y Zonas de Interés Turístico Nacional.

3) Reglamento de los centros no oficiales de enseñanzas turísticas.

4) Establecimientos de zonas para bañistas en el mar y las playas.

5) Clasificación de embalses según su posible aprovechamiento secundario turístico.

6) Ley Reguladora de las Costas Marítimas y Ley de Puertos Deportivos.

7) Regulación de la colaboración entre la Administración turística y el Patrimonio Forestal del Estado.

8) Créditos a corporaciones locales radicadas en zonas turísticas para la realización de obras de interés turístico.

9) Créditos para financiar parcialmente la realización de obras de infraestructura en centros o zonas previamente declarados de interés turístico.

10) Créditos para obras de infraestructura de alojamientos turísticos y de centros de montaña.

Por lo que respecta a la política de demanda hay que señalar que fue escasa y que la Administración se limitó a intentar organizarla administrativamente, a promocionarla y a ejercer un cierto control sobre la misma:

1) Creación del Libro de Interés Turístico y de la Fiesta de Interés Turístico.

2) Reglamentación de las agencias de viajes.

3) Ordenación de los Centros de Iniciativas Turísticas. 
4) Regulación del Registro de Empresas Turísticas Exportadoras.

Por último, en cuanto a las medidas de política turística que podrían calificarse como mixtas merece la pena destacar:

1) Creación del Instituto de Estudios Turísticos.

2) Creación de premios relacionados con el turismo, como los dedicados a periodistas extranjeros, embellecimiento y mejora de los pueblos españoles, estaciones de servicio en carretera, escritores de turismo, estaciones de ferrocarril, diarios y revistas, fotografía turística, entre otros.

\section{La democracia (1975-2000)}

Hubo cambios significativos por lo que respecta a las competencias turísticas y al grado de intervención estatal. La recuperación de la democracia y, sobre todo, la aprobación de la Constitución de 1978 ejercieron una clara influencia sobre los mismos.

El nuevo modelo de Estado autonómico que configuró la Carta Magna exigió notables cambios institucionales en materia turística. El centralismo uniformador del franquismo dio paso a un proceso descentralizador a favor de las Comunidades Autónomas. No hay que olvidar que las transferencias de turismo figuran entre las primeras que se iniciaron $\mathrm{y}$, también, culminaron. Los reales decretos correspondientes cubren un periodo que va desde 1978 a 1984. Por otro lado, otro cambio institucional significativo del periodo fue la adscripción de las competencias turísticas a ministerios con un carácter netamente económico.

Por último, los cambios políticos y de la política económica en general, con el fín de la planificación indicativa, contribuyeron a que la política de la Administración central fuera algo menos intervencionista en materia turística. De las medidas más relevantes del periodo habría que destacar:

1) Apoyo a la exportación de capitales españoles para efectuar inversiones turísticas en el extranjero.

2) Financiación de circulante a empresas turísticas exportadoras.

3) Restricción a la construcción de nuevas plazas de alojamiento en zonas que se consideraban saturadas y el impulso y la potenciación de la modernización de la oferta hotelera, así como su diversificación.

4) Segundo y Tercer Plan de Modernización Hotelera.

5) Ley sobre Protección de las Costas Marítimas Españolas, Reglamento para la Ejecución de la Ley de Costas de 1969 y Reglamento de la Ley de Puertos Deportivos.

6) Declaración de Territorios de Preferente Uso Turístico.

7) Reglamento de la Ley de Espacios Naturales.

8) Privatización de ATESA. 
9) Nuevas ordenaciones de campamentos, de establecimientos hoteleros y de apartamentos turísticos y viviendas turísticas vacacionales.

10) Apoyo a las zonas de influencia socioeconómica de los parques naturales y reservas nacionales de caza.

11) Liberalización de los precios de los establecimientos hoteleros.

Dentro de este punto merece hacer una distinción entre la etapa socialista y la etapa del Partido popular hasta el año 2000. A la hora de analizar la política turística durante la prolongada etapa socialista habría que distinguir dos subperiodos. El primero, que se extiende hasta los últimos años de la década de los ochenta, estuvo muy marcado por la culminación del proceso de transferencias turísticas a las Comunidades Autónomas. Fueron unos años en los que la Administración central fue incapaz de definir una estrategia turística nacional e hizo una cierta dejación de sus funciones, mientras que las Comunidades Autónomas, con competencias exclusivas en materia de turismo, mantuvieron en líneas generales una orientación bastante continuista con relación a la que tradicionalmente había venido siguiendo aquélla. Sin embargo, a lo largo de los años noventa se introdujeron una serie de cambios cualitativos importantes en la organización administrativa turística, se intentó facilitar una mayor coordinación y colaboración entre todos los agentes que intervienen en el turismo y se definieron, por fin, nuevos objetivos estratégicos. Los agentes turísticos, públicos y privados, entendieron que España estaba entrando en un nuevo modelo turístico que requería, hasta cierto punto, reemplazar la estrategia de liderazgo de costes por otra basada en la diferenciación del producto y en la calidad. Competitividad, calidad, eficiencia, diversificación, sostenibilidad, se convirtieron en conceptos claves para la Administración Central y, también, para las diferentes Comunidades Autónomas.

Merecen una especial atención dos estudios e iniciativas que simbolizan la toma de conciencia por parte de los poderes públicos de los cambios que se estaban operando en los mercados turísticos, así como de la necesidad de acompañar procesos de mejora y adaptación de la oferta española a tales cambios, tanto en sus aspectos empresariales como de entorno: el Libro Blanco del Turismo Español y el Plan Marco de Competitividad del Turismo Español (Plan Futures). En el primero, que vio la luz en 1990, se aconsejaba avanzar de forma combinada en tres direcciones:

1) Liderazgo en la relación calidad-precio, especialmente en sol y playa.

2) Diferenciación en segmentos distintos al de sol y playa en los que España pudiera optar a cuotas de mercado razonables y donde no fuera imprescindible un liderazgo de costes.

3) Especialización en aquellos casos en los que existiera poco riesgo de aparición de productos sustitutivos y alta debilidad por parte de los competidores.

El Plan Futures, aprobado en el verano de 1992, representó el primer paso para definir una estrategia turística nacional e iniciar un proceso de colaboración entre el Estado y las Comunidades Autónomas. Los objetivos finales perseguidos eran aumentar la calidad de vida de los agentes involucrados en la actividad turística, conseguir un sector más competitivo y rentable que contribuyera de forma sostenida y equilibrada al crecimiento económico de las zonas turísticas y conservar y mejorar los entornos naturales y culturales. La primera fase el Plan Marco, correspondiente al cuatrienio 19921995, desarrolló cinco grandes planes operativos: 
1) Coordinación y Cooperación Institucional.

2) Modernización e Innovación Turística.

3) Nuevos Productos Turísticos.

4) Promoción, Marketing y Comercialización.

5) Excelencia Turística.

En la segunda fase, la prevista para el periodo 1996-1999, los planes y objetivos fueron los siguientes:

1) Coordinación entre las administraciones públicas y con el sector privado.

2) Mejora de la calidad del producto y servicio turístico español.

3) Mejora de la capacidad tecnológica y de innovación de las empresas del sector turístico.

4) Impulso a la confluencia de actuaciones públicas y privadas para la mejora de la oferta turística de los destinos.

5) Cooperación con los países en desarrollo y apoyo a la internacionalización de las empresas turísticas españolas.

6) Mejora de la cualificación y especialización del capital humano de la industria turística.

7) Profundización en la diversificación y desestacionalización de la oferta turística.

8) Apoyo al conocimiento riguroso del sector y difusión de los resultados.

Por último la política turística del Partido Popular, es decir, los últimos años del siglo pasado no supusieron una ruptura en materia de política turística. Ni en el ámbito estatal ni en el autonómico. Los máximos responsables turísticos profundizaron en la tendencia observada desde comienzos de la década de los años noventa. Buena prueba de ello son las siguientes actuaciones:

1) II Plan Marco de Competitividad y Planes de Excelencia y Dinamización Turística.

2) Plan Estratégico de Actuaciones de la Administración del Estado, en el que se definieron las siguientes acciones concretas a desarrollar: cooperación interadministrativa, promoción y comercialización, estadísticas europeas, Unión Europea, formación turística, competitividad, diversificación, sostenibilidad medioambiental, calidad turística, internacionalización de la empresa turística, actuaciones en destino, medidas de apoyo y Paradores de Turismo.

3) Instituto de la Calidad de la Hostelería Española e Instituto de la Calidad de las Agencias de Viajes.

4) Inicio de los estudios universitarios de turismo. 
5) Presentación, tras la clausura del III Congreso Nacional de Turismo, celebrado en Madrid en noviembre de 1997, de un conjunto de medidas de actuación para llevar a cabo a lo largo del periodo 1998-2000: implantación de un sistema homogéneo de información y señalización turística, realización de un programa de turismo sostenible, apoyo en la Unión Europea al mantenimiento del tipo reducido del IVA, creación del Observatorio del Turismo, potenciación del programa de internacionalización de la empresa turística, implantación de nuevas fórmulas de turismo social, implantación y generalización de la imagen de marca de la calidad turística.

\section{PLANIFICACIÓN TURÍSTICA DE LOS ESPACIOS URBANOS Y COSTES SOCIALES IMPUTABLES AL TURISMO DE GRANDES EVENTOS}

En este apartado nos servirá para empezar a situarnos para el último punto del trabajo en el cual analizaremos tres ejemplos de grandes eventos realizados en el litoral mediterráneo y la transformación urbana que estos han tenido sobre el territorio.

La planificación del turismo en espacios urbanos presenta claras especificidades ya que la integración de la actividad en este medio acontece en el transito de la ciudad industrial a la ciudad de la información, del conocimiento y de los servicios, con una radical transformación en la que, junto con otros procesos (nuevos equipamientos, urbanización difusa, deslocalización de determinados usos), aparecen los grandes centros con funciones de ocio, comercio y deporte (Dachevsky, 2001), además de la organización de eventos que tratan de proyectar la imagen de la ciudad.

El turismo contribuye a la gestación de la ciudad postmoderna en la que junto a operaciones de renovación urbanística, los shopping malls, complejos culturales, lúdico-recreativos y deportivos, junto a los megaeventos, contribuyen a una nueva función de las ciudades, algo que también se ve impulsado por la creación de entidades mixtas de gestión basadas en la co-financiación, la responsabilidad compartida y agilidad en el funcionamiento.

El instrumento básico y más convencional que ha contribuido a ordenar el turismo en las ciudades es el planteamiento urbanístico (clasificación y calificación del suelo, regulación y normas para la edificación, trazado de viario, espacios públicos y equipamientos...) con todas las limitaciones que supone la utilización de una herramienta de planificación no pensada para la ciudad turística ya que este tipo de planeamiento se orienta a objetivos generales del crecimiento urbano y criterios de urbanización, con especial atención a los espacios residenciales y las áreas industriales.

No obstante, son evidentes las limitaciones de los instrumentos de planeamiento urbanístico convencional a la hora de integrar la función turística en las ciudades por lo que el instrumento más apropiado y más utilizado a esta escala es el plan estratégico, aunque su elaboración y puesta en marcha debe coordinarse e incluso su operatividad requiere apoyarse, para la ejecución de un importante número de propuestas, en las formulaciones por la vía del planeamiento urbanístico municipal.

Lo más destacable es la forma en que el turismo se inserta en las políticas urbanas y se materializa en su planificación estratégica y que aún con resultados desiguales, consiguen movilizar en buena parte el tejido socioeconómico y entienden la planificación como componente de la gestión y no como un fin en si mismo. 
A partir de las experiencias de instrumentos de planificación del turismo en ciudades, se observa que, además de una atención especial al citymarketing y a la programación de grandes eventos que contribuyen a la proyección de la imagen y a su posición competitiva, las estrategias impulsadas suelen integrar medidas relativas los siguientes aspectos:

- Dimensión social y cultural del turismo urbano: basadas en el compromiso social y en la colaboración entre agentes, tratando además de utilizar la función turística como factor de cohesión y articulación social de la ciudad. Otras medidas en esta misma dimensión atañen al modo en que el turismo puede contribuir a la valorización de la identidad urbana como referencia.

- Dimensión económica: la multifuncionalidad que acompaña al turismo en la estructura urbana debe apoyarse en el mantenimiento de la actividad comercial y residencial en los barrios y distritos más frecuentados por los turistas así como en políticas de precios acordes con el producto que se oferta $\mathrm{y}$ en tratar de alcanzar un equilibrio entre flujos de turismo y excursionismo y en actuar contra la estacionalidad acusada de la actividad turística. Las iniciativas endógenas son otro aspecto que debe verse favorecido a la hora de las inversiones y la creación de negocios.

- Dimensión ambiental: además de las medidas relativas al control de la contaminación atmosférica y acústica, preocupa también la gestión de los residuos, si bien el tema estrella de las ciudades turísticas, en el aspecto ambiental, es la movilidad interna y la forma de resolver de un modo sostenible los desplazamientos. Soluciones de movilidad sostenible, basadas en diversas iniciativas (peatonalización de vías comerciales, apuesta por el transporte público, aparcamientos disuasorios, red de carriles para bicicletas...) forman parte de planes de ordenación de tráfico y transporte o de los propios planes estratégicos, de cuya gestión depende en buena medida el éxito de la ciudad como destino turístico.

- Dimensión urbanística y territorial: la transformación de las ciudades en destinos de turismo se basa en buena medida en operaciones de renovación urbana y creación de nuevas piezas emblemáticas. Se trata de actuaciones diversas, desde la recuperación de áreas degradadas, pasando por la puesta en marcha de planes de urbanismo comerciales para centros urbanos, hasta la construcción de nuevos complejos y equipamientos lúdico-culturales y comerciales, además de la mejora de las infraestructuras de acceso a la ciudad y la conectividad con áreas emisoras y mercados potenciales de turismo: alta velocidad ferroviaria, mejoras y ampliaciones de los aeropuertos y de sus enlaces, entre otras.

Esta es la teoría y los rasgos generales de la planificación turística de los espacios urbanos y que a continuación pondremos en práctica con el análisis de tres grandes eventos y podremos observar y ver plasmado muchas de las ideas que acabamos de exponer.

\section{LA POLÍTICA DE LOS GRANDES EVENTOS}

Los grandes eventos más significativos y que nos mostraran de manera excelente lo que se ha intentado mostrar. Todo lo expuesto con anterioridad son los antecedentes y la teoría para poder comprender lo que a continuación se va a exponer. 


\section{Análisis de los casos más relevantes y trasfondo de los grandes eventos}

Los casos más relevantes de los grandes eventos celebrados, y que aún se celebran, en la Comunidad Valenciana. Este análisis se hará por separado para desengranar las particularidades de cada caso y como repercute sobre el territorio y sobre la sociedad.

\section{a) Fórmula 1}

Ficha técnica:

\begin{tabular}{|c|c|}
\hline Ciudad de celebración & Valencia \\
\hline Año comienzo celebración & 2008 \\
\hline Año final celebración & $2014 *$ \\
\hline $\begin{array}{c}\text { Coste construcción del circuito y } \\
\text { acondicionamiento urbano de la ciudad }\end{array}$ & 120 millones de euros** \\
\hline Coste canon anual & 30 millones de euros** \\
\hline Rentabilidad económica & 7 millones de euros** \\
\hline
\end{tabular}

* Se está negociando una prórroga del contrato hasta 2021

** Son cifras aproximadas, ya que el gobierno del Partido Popular no muestra el contrato de la Fórmula 1 alegando confidencialidad.

En la ficha técnica de la celebración de la Fórmula 1 se puede observar en algunos casos, aproximados, los gastos que ha tenido y que tiene este gran evento. Si nos ponemos analizar la inversión que se ha hecho es un gasto superfluo, recordemos que Camps (expresidente de la Comunidad Valenciana) y su gobierno se comprometieron a un coste cero, cosa que obviamente como podemos observar no cumplieron. En esta Comunidad se ha priorizado los eventos especulativos en detrimento de los servicios sociales y las necesidades básicas de los ciudadanos. Según Ana Cervera, del grupo de Educación de Democracia Real Ya, "a la ciudadanía le ha costado por ahora 210 millones de euros la Fórmula 1, y cuando se habla de impactos positivos, estos se limitan a puestos de trabajo efímeros de un fin de semana y beneficios en la hostelería de no más de siete días".

Ahora hay que plantearse una pregunta: ¿Es real la imagen vanguardista que se llevan los visitantes de los grandes eventos? Mientras se celebraba el gran premio -explica Cervera- "hay niños en Valencia sin plaza en la enseñanza pública y otros que estudian en barracones; se cierran hospitales dejando grandes áreas de la ciudad sin un centro hospitalario próximo; se eliminan ayudas al alquiler de la vivienda, se aplican recortes en la sanidad pública y la Ley de Dependencia permanece en pañales”.

Esta reflexión que hace Cervera en el párrafo anterior es muy interesante y creo muy acertada. Realmente esa es la situación en la cual se encuentra nuestra Comunidad Valenciana hoy en día, se prioriza el beneficio de unas pocas personas en detrimento de muchas otras personas que se les niega unos servicios mínimos como pueden ser la educación y la sanidad. Podríamos plantearnos ¿Existe el estado de bienestar realmente? Observando estos datos no creo que todos los lugares de la comunidad 
estén llegando el dinero para realizar hospitales y centros estudiantiles, tanto de primaria como de secundaría, no puede ser que vayamos de sociedad desarrollada cuando no lo estamos demostrando y tenemos a una cantidad de alumnos estudiando de una manera tercermundista, son cosas que parecen difícil que puedan pasar en la época que nos encontramos pero que realmente están sucediendo en este mismo momento y no parece que se esté poniendo mucho remedio.

Un pequeño ejemplo lo podemos encontrar muy cerca de donde se sitúa el circuito, en el distrito marítimo de Valencia se puede constatarse la precariedad del estado del bienestar valenciano. Un informe de Democracia Real Ya señala que en el barrio "Camins al Grau" (junto al circuito) hay censados más de 750 niños nacidos en 2005, mientras que sólo se han ofrecido 395 plazas en centros educativos para niños de seis años. En el mismo sector de la ciudad el Plan General reserva tres solares para la construcción de colegios, en los que no se ha dado un solo paso para su ejecución. Lo mismo ocurre en otras áreas de Valencia más alejadas del gran evento, como el popular barrio de Russafa. Tras más de una década de promesas incumplidas, los vecinos continúan esperando la construcción de un colegio (150 niños esperan plaza escolar en Russafa) que ya dispone de licencia de obras y una reserva presupuestaria por parte de la Conselleria de Educación.

En sanidad las necesidades no son menos perentorias. Pese a los dos años de retraso en los plazos para la finalización de las obras, el nuevo hospital de Llíria, que dará servicio a 70.000 habitantes de tres comarcas valencianas, costará 30 millones de euros, la misma cantidad que supuestamente (los contratos permanecen ocultos) paga la Generalitat por el canon anual al magnate Bernie Ecclestone. El mismo día que finalizaba el gran premio, UGT informaba del retraso en el cobro de la paga extraordinaria de 60.000 profesionales sanitarios de hospitales, centros de salud y consultorios valencianos.

Centrarse en los grandes eventos implica unas prioridades en el gasto, la Fórmula 1 por encima de los servicios públicos, y una voluntad política por materializar esta opción. Ejemplos hay muchos. Democracia Real Ya-Valencia ha calculado que la construcción del circuito ha costado aproximadamente lo mismo (120 millones de euros) que las inversiones en equipamientos y nuevas tecnologías para el nuevo hospital universitario La Fe de Valencia (entre otras prestaciones, se anunciaron 35 quirófanos, 1.000 camas y 195 salas de consultas externas).

Joana Martínez subraya que con los 30 millones de euros que anualmente se abonan de canon, podrían construirse un mínimo de cuatro centros educativos públicos de primaria e infantil en un año (incluidos los equipamientos) y otros cuatro institutos públicos de enseñanza secundaria (incluidos gastos de personal, funcionamiento, comedor y transporte en un año).

La ristra de servicios básicos desatendidos sería interminable. El País Valenciano es una de las cinco autonomías que suspende la Ley de la Dependencia y con el dinero del canon podrían -según Democracia Real Ya- concederse más de 200.000 ayudas anuales para personas dependientes. O se podrían dar a 595 investigadores las ayudas de tres años para formación que concede la Conselleria de Educación. O financiar los trabajos de 500 investigadores predoctorales en grandes centros de investigación.

La verdad que las posibilidades que se podrían hacer con todo este dinero son infinitas, lo que se quiere demostrar que el estado del bienestar podría estar bien atendido si se invirtiera el dinero en lo que se tiene que invertir y no hacer esta política de grandes eventos, que no es una inversión de futuro ni genera riqueza sostenible a largo plazo. 
El Circuito donde se celebra este gran evento se llama "Valencia Street Circuit". Son muchas las irregularidades, para empezar no cuenta con declaración de impacto ambiental. El nivel de ruidos está por encima de los umbrales admitidos; no hay estudio de impacto ambiental; está edificado sobre zona inundable... Todo esto cuando hablamos de coches de competición que funcionan a 300 kilómetros por hora, lo que en una actividad industrial dentro de la ciudad habría que considerarla “actividad peligrosa”. Pero no sólo eso. El País Valenciano es uno de los territorios de Europa que más se aleja del cumplimiento del Protocolo de Kyoto y Valencia, una de las ciudades con mayor nivel de contaminación acústica y atmosférica de Europa procedente del tráfico privado.

Ante tantas ilegalidades, ¿Cómo ha salido adelante el proyecto de obras? El Proyecto se aprueba mediante un "Decreto de Excepcionalidad" por un supuesto "interés general", con fecha de 8 de febrero de 2008. Eso les ha permitido saltarse hasta 8 leyes diferentes. Las obras del circuito no figuran como tales, sino como "Actuaciones de prolongación de la Avenida Francia y la Alameda", y su conexión con el puerto.

Y todas estas claves aún se acentúan más cuando se observa que en la provincia de valencia ya existe un circuito en Cheste. De hecho, el circuito de Cheste para carreras de coches y motos se encuentra a menos de 30 kilómetros y acumula unas pérdidas de 60 millones de euros desde que se inauguró hace una década. No se entiende este despilfarro en algo que tanto va a perjudicar a la ciudad y molestar a los ciudadanos. El Gobierno Valenciano vendió a la opinión pública que las actividades de la Fórmula 1 ocuparían el circuito únicamente tres días al año, y que el resto sería de uso recreativo. Pero la realidad es que se encuentra vallado y cerrado al público durante todo el año.

\section{b) Copa del América}

Ficha Técnica:

\begin{tabular}{|c|c|}
\hline Ciudad de celebración & Valencia \\
\hline Año asignación & 2003 \\
\hline Años de celebración & 2007 y 2009 \\
\hline Coste construcción instalaciones & 900 millones de euros* \\
\hline Rentabilidad económica & Sin cifras concretas** \\
\hline
\end{tabular}

*Son cifras aproximadas, ya que no existen cifras oficiales.

**No hay datos de una estimación de beneficios concretos.

En este segundo gran evento que vamos a analizar es el de la Copa del América que se celebró en las ediciones de 2007 y 2009 en la ciudad de Valencia, el año de asignación de este gran evento fue el 2003, y a partir del cual empezaron todas las obras.

Hay que plantearse, ¿Qué hay detrás de la Copa del América? Para responder a esta pregunta Emilio Martín Estudillo, dice que la respuesta es simple: especulación salvaje barnizada de deporte y glamour. Y puede ser que se piense lo mismo después de ver las consecuencias sobre la sociedad y las consecuencias sobre el territorio que tuvo. 
Los promotores de la Copa del América, proyectaron la remodelación del puerto, basada en la creación de un canal navegable que conecta la dársena interior del puerto con mar abierto, con el objeto de facilitar la entrada y salida de la veintena de embarcaciones que participaron en la regata. El impacto más destacable de esta actuación fue el basculamiento de la playa de la Malvarrosa, que sufrirá una regresión de 20 metros provocada por el dique que permite el adentramiento del citado canal varios cientos de metros en el mar. Para subsanar esta pérdida se propone la regeneración de la playa con una cantidad de 113.000 a 500.000 metros cúbicos de arena durante un periodo que oscila entre los 12 y los 20 años. El dique provocará, además, un gran impacto paisajístico al recortar considerablemente la visión del horizonte desde la Malvarrosa. Otras consecuencias del cambio en la dinámica litoral ocasionado por esta obra será la desaparición de nueve caladeros de pesca y la más que probable afección de las playas situadas más al sur, ya en pleno Parque Natural de La Albufera.

Sin salir del entorno portuario, exactamente entre el muelle de Poniente y el muelle del Turia, hallamos la primera gran maniobra especulativa que esconde esta obra especulativa del puerto y que contempla la recalificación para uso residencial de varias parcelas de propiedad estatal. Este cambio de uso viene dado por la necesidad de encontrar financiación a las obras derivadas del evento, lo que ha llevado a los promotores a proyectar la venta de suelo portuario después de la celebración del evento deportivo. Este suelo acogerá 4.000 viviendas de alto standing, muchas de ellas con amarres incluidos. Las viviendas se encontrarán situadas junto a la dársena interior, destinada ha convertirse en puerto deportivo para embarcaciones de lujo.

Si se habla de la repercusión que han acaparado los barrios colindantes, el más perjudicado es el de El Cabanyal. Barrio venía manteniendo una batalla de años con el Ayuntamiento de Valencia para evitar la demolición de 943 viviendas a las que la pala excavadora echaría abajo para conectar el centro de Valencia con el entorno portuario a través de la ampliación de la Avenida Blasco Ibáñez. Este barrio, calificado como Bien de Interés Cultural por su particular trazado y arquitectura, recibió como un jarro de agua fría el fallo del Tribunal Superior de Justicia de la Comunidad Valenciana que daba vía libre al proyecto municipal, poco tiempo después de que la Jarra de las Cien Guineas llegara a Valencia. A nadie se le escapa el gran movimiento especulativo que recorrerá la elongación de Blasco Ibáñez una vez "saneado" el barrio de buena parte de sus habitantes -en su mayoría personas mayores o de rentas bajas- expulsados bien por la piqueta, bien por el proceso de abandono sufrido por el barrio, eterno olvidado de la administración local.

Si bien El Cabanyal es el más afectado por este terremoto especulador revestido de barniz deportivo, tampoco se librarán los deprimidos poblados marítimos colindantes con el puerto, como la Malvarrosa, Natzaret y las zonas de huerta cercanas; hemos de recordar que el puerto ya experimentó una ampliación considerable, la Zona de Actuación Logística (ZAL), que supuso la desaparición de valiosas zonas de huerta y parte de sus poblados (La Punta). Incluso el resto de vecinos y vecinas de Valencia se verá perturbado, si damos crédito a lo que se admite en numerosos foros: un importante incremento en los precios de viviendas, alquiler, servicios y transporte público, ligado, claro está, al advenimiento de la Copa.

Este incremento del coste de la vida no fue asociado a una mejora en la calidad de la misma de los ciudadanos y ciudadanas de Valencia a los que apenas se les prometió 10.000 puestos de trabajo de carácter temporal, o al menos así lo reconocen los estudios de impacto realizado hasta la fecha. Tampoco fue muy agradable para numerosos habitantes de la ciudad el incremento de la presión policial vinculada a la seguridad del evento, que ya se dejó notar en las preregatas, impidiendo manifestaciones críticas con el evento. También son previsibles estrategias de "limpieza social" como 
las realizadas en torno a otros eventos en otros lugares del estado y que en Valencia comenzaron con campañas de tolerancia cero en barrios como el de Russafa, caracterizado por su pluralidad étnica.

A pesar de todas estas repercusiones hay que hacer otra distinción más que es la situación en la cual se encuentran las instalaciones, que en estos momentos están obsoletas y se están empezando a degradar por su obsoleto uso, una muy buena comparación puede ser con las instalaciones de la Exposición Universal en Sevilla en 1992. Esto no debería ser de esta manera y ya que se ha hecho esta barbaridad urbanística se debería un uso para estas instalaciones que favoreciera a la sociedad y fuera un uso común para todos y no solo para unos cuantos.

\section{c) Fòrum de les Cultures}

Ficha técnica:

\begin{tabular}{|c|c|}
\hline Ciudad de celebración & Barcelona \\
\hline Año celebración & 2004 \\
\hline Año asignación & 1996 \\
\hline Coste construcción del recinto & 324 millones de euros \\
\hline Visitantes esperados & 5 millones de personas \\
\hline Visitantes contabilizados & 3.323 .120 personas \\
\hline
\end{tabular}

Se puede decir que el Fòrum Universal de les Cultures fue el resultado de una propuesta, un tanto difusa, surgida del Ayuntamiento de Barcelona en octubre de 1996 y realizado en 2004.

Los grupos contrarios consideraban que el Fòrum había sido un proyecto especulativo, que banalizaba la cultura, favorecía a los poderes económicos y transformaba la ciudad en un centro especializado en ocio, con la temporalización y dependencia coyuntural que este hecho conlleva. Y es que centrándonos en el espacio construido, tema del artículo, existió desde un principio una diferencia de percepción respecto a cuales eran las transformaciones físicas que el Fòrum, por sí mismo, generaba. Oficialmente, el recinto Fòrum se limitaba al espacio que ocupó la celebración del evento (30 hectáreas). En dicho espacio, dividido entre los términos municipales de Barcelona y Sant Adrià del Besòs y anteriormente ocupado por una depuradora y terrenos sin un uso definido, se construyó, en parte en terrenos ganados al mar, un gran centro de convenciones, un puerto deportivo, distintos parques, una playa artificial y una gran explanada que cubre la depuradora. El único equipamiento privado de la zona fue un hotel, construido de forma adosada al centro de congresos. La gestión del puerto y el centro de convenciones corren actualmente a cargo de agentes privados.

El evento Fòrum Universal de les Cultures habría podido celebrarse en otras ubicaciones, pero ya desde el principio se priorizaron los terrenos del Besòs. La idea no era nueva. Entre otros, el plan Cerdà preveía un gran parque en aquella zona y el plan Macià proyectaba recuperar el litoral. Pero ninguno de estos grandes proyectos se había materializado y la zona había sido, históricamente, el "trastero" de Barcelona: un campo de chabolismo hasta los años setenta, depuradora, incineradora, central térmica... En el momento de presentar el proyecto del Fòrum el área estaba en pleno proceso 
de transformación. La industria, que tradicionalmente había ocupado el espacio, se estaba trasladando a otros emplazamientos y los terrenos que había ocupado el ferrocarril estaban en desuso desde los grandes cambios realizados con motivo de los Juegos Olímpicos. Por lo tanto, la zona disponía de un potencial importantísimo en terrenos, no hay que olvidarlo, mayoritariamente públicos.

El primer gran conflicto de carácter urbanístico, aún fuera del período estudiado pero interesante de señalar, fue el relacionado con qué proyecto realizar. En 1996 se preveía una actuación bastante diferente a la que finalmente se construyó: no se cubría la depuradora y todas las actividades se realizaban en una gran plataforma de 35 hectáreas ganada al mar. Ahora bien, el 7 de septiembre de 1999 la Dirección General de Costas emitió un informe desfavorable a la modificación de la línea litoral. El consorcio encargado de las obras rebatió el informe, pero el 11 de abril de 2000, el Ministerio de Medio Ambiente reafirmó la prohibición, calificando la operación de "maniobra de carácter marcadamente mercantil" y de "privatización encubierta del dominio público maritimoterrestre".6 Finalmente, el 19 de junio se llegó a un acuerdo político entre el ministro de Medio Ambiente, en aquel momento Jaume Matas, y el alcalde de Barcelona, Joan Clos, por el que el Ayuntamiento modificaba el proyecto y asumía el coste de posibles regeneraciones de las playas.

Evidentemente hay que analizar lo acontecido en los barrios colindantes al recinto del Fòrum de les Cultures, es verdad que se emprendieron acciones para sanear los barrios, pero las quejas de los vecinos eran que no llegaban de la misma manera que al recinto del gran evento. Hay que hacerse la pregunta ¿Hubieran llegado las mismas ayudas si no se hubiera realizado este gran evento? Realmente mi opinión y por las informaciones que se han podido observar no hubieran sido las mismas ayudas. Todas estas acciones para sanear el barrio de la Mina no eran más que acciones de saneamiento para dejarlo con una buena imagen para el público asistente al Fòrum, ya que el barrio de la Mina es un barrio con un gran número de chabolas que necesitaba acciones desde hace mucho tiempo.

La mayor pretensión del ayuntamiento de Barcelona con este gran evento, a parte de todo lo que hemos analizado, era la llegada de la diagonal al Mar. Se ha podido demostrar que hay actuaciones que no están muy claras, que primero fueron detenidas por el ministerio de medio ambiente y luego dieron el visto sabiendo de todas las consecuencias medioambientales que traía consigo la celebración de este gran evento.

\section{CONCLUSIONES}

Para concluir este trabajo en el cual hemos hecho un estudio sobre la repercusión política de los grandes eventos y la repercusión sobre el territorio y sobre la sociedad. Se ha podido observar mediante la evolución durante todo el siglo XX el camino hacía la actualidad, era necesario e imprescindible hacer esta evolución porque muchas de las actuaciones acontecidas durante el siglo pasado son la evolución de lo que tenemos hoy en día en esta materia.

Pero realmente el fin que se buscaba y que se ha podido demostrar es la repercusión de los grandes eventos y su trasfondo. Hemos analizado tres solamente de los muchos que hay por analizar, pero los analizados no han sido escogidos al azar, son muy significativos y que demuestran muy bien los fines que tienen estas celebraciones. Se puede observar como estos eventos no benefician a la sociedad, tienen muchos más efectos negativos que positivos, y eso no se puede permitir que por una celebración de esta índole un barrio colindante y necesitados de la ciudad se queden sin ayudas necesarias que llevan pidiendo muchos años y que no haya dinero porque se ha destinado para los 
grandes eventos, y que después los beneficios no son para los ciudadanos, sino para los agentes privados que son los que tienen la competencia de la organización.

Todo lo analizado nos lleva a pensar que los políticos sacan beneficios directa o indirectamente por la celebración de estos grandes eventos, se gastan el dinero de todos en cosas de estas y luego observamos como el estado de bienestar se encuentra en un estado lamentable y apunto de entrar en la quiebra, no es lícito lo que están haciendo y no se les tiene que permitir estas actuaciones. Por otro lado tampoco es licito que se esconda debajo de un gran evento una actuación urbanística que no se permitiría sin esconderse y con acuerdos sospechosos entre las administraciones.

Una muestra de que estas celebraciones no son beneficiosas las encontramos en la Comunidad Valenciana la cual ha sido la primera comunidad autónoma que ha tenido que ser intervenida por la tesorería por no poder afrontar gastos. Y aún dicen que van a continuar con esta política, ¿A dónde quieren llegar? No se hasta cuando podrán aguantar con ello, el ex presidente Camps ya está sentado en los banquillos, si sigue de esta manera alguno más se sentara en el banquillo de los acusados.

Después de todo lo expuesto, poco queda por decir, seria necesario que este tema se estudiara con más profundidad, pues las cuestiones aquí tratadas motivan mucho y variados frentes que merecen ser estudiados.

\section{BIBLIOGRAFÍA}

CALS, J. Turismo y política turística en España: una aproximación. Barcelona: Ariel, 1974. 285 p.

RIVAS GARCÍA, J. Planificación turística. Oviedo: Septem, 2006. 151 p.

VELLAS, F. Economía del turismo internacional. Madrid: Sintesis, 2004. 302 p.

VERA, J. F. LÓPEZ PALOMEQUE, F. MARCHENA, M. J. ANTON CLAVÉ, S. Análisis territorial del turismo y planificación de destinos turísticos. Valencia: Tirant lo Blanch, 2011. 473 p.

VILÁ, J. Introducción al estudio teórico de la geografía. Barcelona: Ariel, 1983. 377 p.

\section{RECURSOS ELECTRÓNICOS}

<http://www.rebelion.org/noticia.php?id=128342>. [25/09/12].

<http://www.rebelion.org/noticia.php?id=73557>. [25/09/12].

<http://www.rebelion.org/noticia.php?id=131716 >. [25/09/12].

<http://www.fundacioncajamar.es/mediterraneo/revista/me0513.pdf > . [25/09/12].

<http://www.elpais.com/articulo/Comunidad/Valenciana/crisis/tumba/rentabilidad/turistica/grandes/e ventos/elpepiespval/20100208elpval_6/Tes>. [25/09/12].

$\langle\underline{\text { http://www.revistamiralls.es/?p=2710 }}\rangle$. [25/09/12]. 
<http://www.elpais.com/edigitales/entrevista.html?id=2820 > . [25/09/12].

<http://www.cev.es/web-

cev/WCEV.nsf/\%28All\%29/734E5BD65576B675C12576C4003953F3/\$File/prensa0812_149.pdf>. [25/09/12].

<http://www.rebelion.org/noticia.php?id=45697>. [25/09/12].

<http://www.rebelion.org/noticia.php?id=14827>. [25/09/12].

<http://www.rebelion.org/noticia.php?id=45697>. [25/09/12].

<http://www.rebelion.org/noticia.php?id=14827>. [25/09/12].

(c) Copyright Dimas Pla Sirvent, 2013.

(c) Copyright GeoGraphos, 2013.

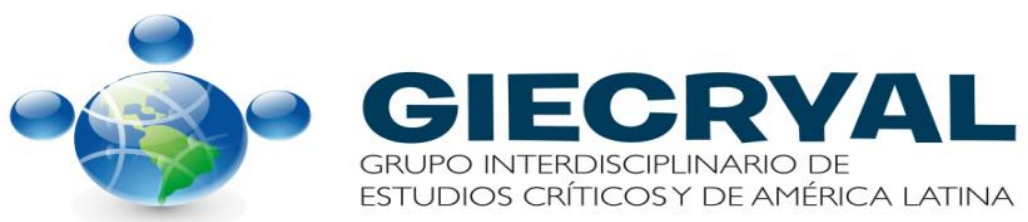

